\title{
DESCRIPTIVE COMPLEXITY OF FUNCTION SPACES
}

BY

\author{
D. LUTZER ${ }^{1}$, J. VAN MILL AND R. POL ${ }^{2}$
}

\begin{abstract}
In this paper we show that $C_{\pi}(X)$, the set, of continuous, realvalued functions on $X$ topologized by the pointwise convergence topology, can have arbitrarily high Borel or projective complexity in $\mathbf{R}^{X}$ even when $X$ is a countable regular space with a unique limit point. In addition we show how to construct countable regular spaces $X$ for which $C_{\pi}(X)$ lies nowhere in the projective hierarchy of the complete separable metric space $\mathbf{R}^{X}$.
\end{abstract}

1. Introduction. Let $C_{\pi}(X)$ be the set of continuous, real-valued functions on a space $X$ and topologize $C_{\pi}(X)$ as a subspace of the full product $\mathbf{R}^{X}$. In [DGLvM] it is shown that if $X$ is completely regular, then $C_{\pi}(X)$ cannot be a $G_{\delta^{-}}, F_{\sigma^{-}}$or $G_{\delta \sigma^{-}}$-subset of $\mathbf{R}^{X}$ unless $X$ is discrete and that for any countable metrizable space $X, C_{\pi}(X)$ will be an $F_{\sigma \delta}$-subset of $\mathbf{R}^{X}$. In the terminology of $[\mathbf{K M}$ and $\mathbf{K}], C_{\pi}(X)$ cannot have multiplicative class 1 and cannot have additive class 1 or 2 , but may have multiplicative class 2 .

In this paper we study the descriptive complexity of $C_{\pi}(X)$ in $\mathbf{R}^{X}$ when $X$ is countable (so that $\mathbf{R}^{X}$ is a complete separable metric space). Our main results can be summarized as follows.

THEOREM. (a) Given any $\alpha<\omega_{1}$, there is a countable regular space $X$ such that $C_{\pi}(X)$ is a Borel subset of $\mathbf{R}^{X}$ having additive class $\beta$, where $\alpha \leq \beta \leq 3+\alpha+2$ $(\S \S 2$ and 3$)$.

(b) Given any $n \geq 1$ there is a countable regular space $Y$ such that $C_{\pi}(Y) \in$ $\mathcal{L}_{n}\left(\mathbf{R}^{Y}\right)-\mathcal{L}_{n-1}\left(\mathbf{R}^{Y}\right)$, where $\mathcal{L}_{n}\left(\mathbf{R}^{Y}\right)$ is the family of projective sets of class $n$ in the complete separable metric space $\mathbf{R}^{Y}(\S 4)$.

(c) There is a countable regular space $Z$ such that $C_{\pi}(Z) \notin \bigcup\left\{\mathcal{L}_{n}\left(\mathbf{R}^{Z}\right): 0 \leq n<\right.$ $\omega\}(\S \S 4$ and 5$)$.

The spaces $X, Y$ and $Z$ in the above Theorem can be obtained from a single general construction which associates with each subset $S \subset 2^{\omega}$ a certain countable regular space $\Sigma_{S}$ having a unique nonisolated point. The descriptive complexity of $S$ in $2^{\omega}$ determines the complexity of $C_{\pi}\left(\Sigma_{S}\right)$ in $\mathbf{R}^{\Sigma_{S}}$. To describe $\Sigma_{S}$ precisely, we begin by letting $T_{n}=2^{n}$ be the set of functions from $\{0,1, \ldots, n-1\}$ into $\{0,1\}$, i.e., the set of ordered $n$-tuples of 0 's and 1's. Let $T=\bigcup\left\{T_{n} \mid n \geq 1\right\}$ and partially order $T$ by function extension. A branch of $T$ is a maximal linearly ordered subset of $T$, i.e., a linearly ordered subset $B \subset T$ having $\operatorname{card}\left(B \cap T_{n}\right)=1$ for each $n \geq 1$. Observe that if $B$ and $\hat{B}$ are distinct branches of $T$, then $B \cap \hat{B}$ must be a finite set.

Received by the editors August 3, 1984.

1980 Mathematics Subject Classification. Primary 03E15, 04A15, 54H05; Secondary 54C35.

${ }^{1}$ Partially supported by NATO grant 1927, NSF grant MCS80-016617 and by Vrije Universiteit, Amsterdam.

${ }^{2}$ Partially supported by Vrije Universiteit, Amsterdam. 
Given $x \in 2^{\omega}$, the set $B_{x}=\{\langle x(0)\rangle,\langle x(0), x(1)\rangle,\langle x(0), x(1), x(2)\rangle, \ldots\}$ is a branch of $T$. Conversely, each branch $B$ of $T$ has the form $B=B_{x}$ for a unique $x \in 2^{\omega}$. Let $B=\{B \mid B$ is a branch of $T\}$.

Let $P(T)=\{A \mid A \subset T\}$ and topologize $P(T)$ using open sets of the form $[Y, N]=\{A \in \mathcal{P}(T) \mid Y \subset A \subset T-N\}$, where $Y$ and $N$ are arbitrary finite subsets of $T$. The resulting space is compact and metrizable, and is homeomorphic to the product space $2^{T}$ under the mapping which identifies each subset $A \in P(T)$ with its characteristic function $\chi_{A}$. The mapping $x \rightarrow B_{x}$ is easily seen to be a homeomorphism of $2^{\omega}$ into $P(T)$ whose image is exactly the set $B$ defined above.

For each subset $S \subset 2^{\omega}$, the collection $\left\{T-\left(B_{x_{1}} \cup \cdots \cup B_{x_{n}} \cup F\right) \mid n \geq 1, x_{i} \in S\right.$ and $F \subset T$ is a finite set $\}$ is a filter base. Let $p_{S}$ be the filter generated by that filter base. Let $\infty$ be any point not in $T \cup 2^{\omega}$ and let $\Sigma_{S}=T \cup\{\infty\}$. Topologize $\Sigma_{S}$ by isolating each point of $T$ and by using the family $\left\{P \cup\{\infty\} \mid P \in p_{S}\right\}$ as a neighborhood base at $\infty$. The space $\Sigma_{S}$ is countable, regular and (since $p_{S}$ is a free filter) is $T_{1}$. The spaces mentioned in the above Theorem are all of the form $\Sigma_{S}$ for various subsets $S$ of $2^{\omega}$.

However, even though the function spaces $C_{\pi}\left(\Sigma_{S}\right)$ for $S \subset 2^{\omega}$ provide enough pathology to prove our Theorem, they are all well behaved in some senses. In $\S 5$ we prove that each $C_{\pi}\left(\Sigma_{S}\right)$ is a Baire Property subset of $\mathbf{R}^{\Sigma_{S}}$ and is meagre in $\mathbf{R}^{\Sigma_{S}}$ (equivalently, $C_{\pi}\left(\Sigma_{S}\right)$ is not a Baire space) and we exhibit a countable regular space $X$ with a unique nonisolated point such that $C_{\pi}(X)$ is a second category subset of $\mathbf{R}^{X}$ (equivalently, $C_{\pi}(X)$ is a Baire space), is not a Baire Property subset of $\mathbf{R}^{X}$, and is not a Borel, analytic or co-analytic subset of $\mathbf{R}^{X}$ (see Example 5.5).

The standard references for descriptive theory in complete separable metric spaces are [K and $\mathbf{K M}]$. Our topological terminology is consistent with [E] and $\left[\mathbf{O x}_{2}\right]$ is a good source for properties of Baire spaces. The authors wish to thank Jean Calbrix and Fons van Engelen for their comments on an earlier version of this paper.

\section{A lower bound for the complexity of $C_{\pi}\left(\Sigma_{S}\right)$.}

2.1 THEOREM. Let $S \subset 2^{\omega}$ and let $\Sigma=\Sigma_{S}$. Then $C_{\pi}(\Sigma)$ contains a relatively closed subset which is homeomorphic to $S$.

PROOF. Recall that in $\Sigma_{S}$, the point $\infty$ has a neighborhood base consisting of all sets of the form $\{\infty\} \cup\left(T-\left(B_{x_{1}} \cup B_{x_{2}} \cup \cdots \cup B_{x_{n}} \cup F\right)\right)$, where $x_{i} \in S$ and $F \subset T$ is finite. For each $x \in 2^{\omega}$ define a function $f_{x}: \Sigma \rightarrow \mathbf{R}$ by $f_{x}(\infty)=0, f_{x}(t)=0$ if $t \in T-B_{x}$ and $f_{x}(t)=1$ if $t \in B_{x}$. Define $\lambda: 2^{\omega} \rightarrow \mathbf{R}^{\Sigma}$ by $\lambda(x)=f_{x}$. Clearly $\lambda$ is 1-1 and continuous, so that $\lambda$ embeds $2^{\omega}$ as a closed subspace of $\mathbf{R}^{\Sigma}$. Furthermore $\lambda(x) \in C_{\pi}(\Sigma)$ whenever $x \in S$ because for such an $x$, the function $f_{x}$ is constant on the neighborhood $\{\infty\} \cup\left(T-B_{x}\right)$ of $\infty$. Conversely, if $f_{x} \in C_{\pi}(\Sigma)$ for some $x \in 2^{\omega}$, then $f_{x}^{-1}\left[\left(-\frac{1}{2}, \frac{1}{2}\right)\right]$ must be a neighborhood of $\infty$ so that for some $x_{1}, \ldots, x_{n} \in S$ and some finite $F$, the set $f_{x}^{-1}\left[\left(-\frac{1}{2}, \frac{1}{2}\right)\right]=\{\infty\} \cup\left(T-B_{x}\right)$ must contain the basic neighborhood $\{\infty\} \cup\left(T-\left(B_{x_{1}} \cup \cdots \cup B_{x_{n}} \cup F\right)\right)$. But then $B_{x} \subset B_{x_{1}} \cup \cdots \cup B_{x_{n}} \cup F$ so that $B_{x_{i}} \cap B_{x}$ is infinite for some $i$ and hence $B_{x}=B_{x_{i}}$, i.e., $x=x_{i} \in S$. Therefore $\lambda[S]=C_{\pi}(\Sigma) \cap \lambda\left[2^{\omega}\right]$ showing that $\lambda[S]$ is a relatively closed subset of $C_{\pi}(\Sigma)$.

2.2 COROLlaRY. If $S$ is not a Borel subset of $2^{\omega}$ (resp., if $S$ is not a projective subset of $2^{\omega}$ ), then $C_{\pi}\left(\Sigma_{S}\right)$ is not a Borel subset (resp. a projective subset) of $\mathbf{R}^{\Sigma_{S}}$. 
PROOF. Write $\Sigma=\Sigma_{S}$. In the complete separable metric space $\mathbf{R}^{\Sigma}$, a relatively closed subset of a Borel (resp., projective) set is again a Borel (resp., projective) set in $\mathbf{R}^{\Sigma}$ and it is known that homeomorphisms preserve Borel (resp., projective) sets [K, Chapter 3, §35, IV, Corollary 1 and Chapter 3, §38, VII, Theorem 1] contrary to our assumption that $S$ is not Borel (resp., projective) in $2^{\omega}$.

2.3 COROLlaRY. There is a countable regular space $X$ such that $C_{\pi}(X)$ is not a Borel subset of $\mathbf{R}^{X}$.

Proof. Let $S$ be a non-Borel subset of $2^{\omega}$ and let $X=\Sigma_{S}$. Now apply 2.2.

3. An upper bound for the Borel complexity of $C_{\pi}\left(\Sigma_{S}\right)$. In $\S 2$ we proved that $C_{\pi}\left(\Sigma_{S}\right)$ always contains a closed subspace homeomorphic to $S$ so that if $S$ is not a Borel set, then neither is $C_{\pi}\left(\Sigma_{S}\right)$. In this section we study the situation where $S$ is a Borel subset of $2^{\omega}$ and we prove

3.1 THEOREM. Let $S$ be a Borel subset of $2^{\omega}$ having additive class $\alpha \geq 1$ and let $\Sigma=\Sigma_{S}$. Then $C_{\pi}(\Sigma)$ is a Borel subset of $\mathbf{R}^{\Sigma}$ of class $\beta$, where $\alpha \leq \beta \leq 3+\alpha+2$.

PROOF. Following the notation of $\S 1$, we let $p=p_{S}$ be the filter on $T$ generated by all sets of the form $T-\left(B_{x_{1}} \cup \cdots \cup B_{x_{n}} \cup F\right)$, where $x_{j} \in S$ for $1 \leq j \leq n$ and $F$ is any finite subset of $T$.

For each $m \geq 1$, define $\psi_{m}: \mathbf{R}^{\Sigma} \rightarrow \mathcal{P}(T)$ by $\psi_{m}(f)=\{t \in T|| f(\infty)-f(t) \mid \geq$ $1 / m\}$. In Lemma 3.2 we show that $\psi_{m}$ is a Borel mapping of class 1 . Next, define a set $D \subset \mathcal{P}(T)$ by $D=\{A \in \mathcal{P}(T) \mid A \cap P=\varnothing$ for some $P \in p\}$. In Lemma 3.6 we prove that $D$ is a Borel subset of $P(T)$ of additive class $\leq 2+\alpha$ so that $\psi_{m}^{-1}[D]$ is a Borel set of additive class $\leq 3+\alpha$. Because a function $f \in \mathbf{R}^{\Sigma}$ is continuous if and only if $\{t \in T|| f(\infty)-f(t) \mid<1 / m\}$ belongs to $p$ for each $m$, we have $C_{\pi}(\Sigma)=\bigcap\left\{\psi_{m}^{-1}[D] \mid m \geq 1\right\}$ showing that $C_{\pi}(\Sigma)$ is a Borel set of additive class $\beta \leq(3+\alpha+2)$.

From $\S 2$, a closed subspace of $C_{\pi}(\Sigma)$ is homeomorphic to $S$, so the additive class of $C_{\pi}(\Sigma)$ cannot be smaller than the additive class of $S$ and we obtain $\alpha \leq \beta$.

All that remains is to prove some lemmas.

\subsection{LEMMA. Each $\psi_{m}$ is a Borel map of class 1 .}

Proof. It is enough to show that $\psi_{m}^{-1}[[Y, N]]$ is an $F_{\sigma}$-subset of $\mathbf{R}^{\Sigma}$ for each basic open set $[Y, N]$ in $\mathcal{P}(T)$. Now

$$
\begin{aligned}
\psi_{m}^{-1}[[Y, N]]= & \left\{f \in \mathbf{R}^{\Sigma} \mid Y \subset\{t \in T|| f(t)-f(\infty) \mid \geq 1 / m\}\right\} \\
& \cap\left\{f \in \mathbf{R}^{\Sigma} \mid\{t \in T|| f(t)-f(\infty) \mid \geq 1 / m\} \subset T-N\right\} .
\end{aligned}
$$

The first of those two sets is closed and, since $N$ is finite, the second is open. Hence their intersection is an $F_{\sigma}$-set, as claimed.

3.3 LEMma. The set $A=\left\{A \in P(T) \mid\right.$ for some $B_{1}, \ldots, B_{n} \in B$ and some finite $\left.F \subset T, A \subset B_{1} \cup \cdots \cup B_{n} \cup F\right\}$ is a $\sigma$-compact subset of $P(T)$.

PROOF. For a fixed finite $F \subset T$ and a fixed $n$, let $A(F, n)=\left\{\left(A, B_{1}, \ldots, B_{n}\right) \mid B_{i}\right.$ $\in B$ and $\left.A \subset B_{1} \cup \cdots \cup B_{n} \cup F\right\}$. Then $\mathcal{A}(F, n)$ is a closed subset of the compact space $P(T) \times B^{n}$. Let $\pi_{n}: P(T) \times B^{n} \rightarrow \mathcal{P}(T)$ denote first coordinate projection. Then $\mathcal{A}=\bigcup\left\{\pi_{n}[\mathcal{A}(F, n)]: n \geq 1\right.$ and $F \subset T$ is finite $\}$ so that $\mathcal{A}$ is a $\sigma$-compact subspace of $\mathcal{P}(T)$ as claimed. 
3.4 Notation. Recall that $B$ is the set of all branches of $T$, topologized as a subspace of the compact metric space $P(T)$. Being the continuous image of $2^{\omega}$ under the map $\mu(x)=B_{x}, B$ is compact. For $n \geq 1$, let $\Phi_{n}=\{\mathcal{K} \subset B \mid \operatorname{card}(\mathcal{K})=n\}$ and let $\Phi=\bigcup\left\{\Phi_{n} \mid n \geq 0\right\}$. Topologize $\Phi$ with the Vietoris topology, i.e., by using all subsets of $\Phi$ of the forms $\{K \in \Phi \mid \mathcal{K} \subset \mathcal{U}\}$ and $\{K \in \Phi \mid K \cap \mathcal{V} \neq \varnothing\}$ as a subbase where $\mathcal{U}$ and $\mathcal{V}$ are arbitrary open subsets of $B$. Then $\Phi$ is a $\sigma$-compact metrizable space [KM, p. 392]. Recall that each branch of $T$ is of the form $B_{x}$ for some $x \in 2^{\omega}$ and let $\Phi_{S}=\left\{\mathcal{K} \in \Phi \mid \mathcal{K} \subset\left\{B_{x} \mid x \in S\right\}\right\}=\left\{\mathcal{K} \mid \mathcal{K}\right.$ is a finite subset of $\left.\left\{B_{x} \mid x \in S\right\}\right\}$.

3.5 LEMma. With $A$ as in 3.3, for each $A \in A$ let $i(A)=\{B \in B \mid B \cap A$ is infinite $\}$. Then $i: A \rightarrow \Phi$ is a Borel mapping of class 2 .

Proof. Fix $A \in A$ and choose branches $B_{1}, \ldots, B_{n}$ and a finite set $F$ with $A \subset B_{1} \cup \cdots \cup B_{n} \cup F$. If $B$ is any branch of $T$ such that $A \cap B$ is infinite, then $B \cap B_{k}$ is infinite for some $k=1,2, \ldots, n$ so that $B$ is one of the branches $B_{1}, \ldots, B_{n}$. Hence $i(A)$ is finite so $i(A) \in \Phi$. (If $A$ is finite, then $i(A)=\varnothing \in \Phi$.)

(a) Fix an open subset $\mathcal{U}$ of $B$ and consider $i^{-1}[\{\mathcal{K} \in \Phi \mid \mathcal{K} \subset \mathcal{U}\}]=\{A \in$ $A \mid i(A) \subset \mathcal{U}\}$. Because $\mathcal{U}$ is an open subset of the compact metric space $B, \mathcal{U}$ is $\sigma$ compact. According to 3.3 , so is $A$, and we conclude that the product space $A \times U^{n}$ is $\sigma$-compact for each $n \geq 1$, where $\mathcal{U}^{n}$ is the product of $n$ copies of $\mathcal{U}$. Fix $n \geq 1$ and fix a finite set $F \subset T$. Then the set $C(n, F)=\left\{\left(A, B_{1}, \ldots, B_{n}\right) \in A \times \mathcal{U}^{n} \mid A \subset\right.$ $\left.B_{1} \cup \cdots \cup B_{n} \cup F\right\}$ is closed in $A \times \mathcal{U}^{n}$, so $C(n, F)$ is $\sigma$-compact. Let $\pi_{n}: A \times U^{n} \rightarrow A$ be first coordinate projection. Then $i^{-1}[\{\mathcal{K} \in \Phi \mid \mathcal{K} \subset \mathcal{U}\}]=\bigcup\left\{\pi_{n}[\mathcal{C}(n, F)] \mid n \geq 1\right.$ and $F \subset T$ is finite $\}$ so $i^{-1}[\{K \in \Phi \mid \mathcal{K} \subset U\}]$ is a $\sigma$-compact subset of $A$ (and therefore a $G_{\delta \sigma}$-subset of $\AA$ ).

(b) Next consider $i^{-1}[\{K \in \Phi \mid K \cap \mathcal{V} \neq \varnothing\}]$, where $\mathcal{V}$ is a compact, open subset of $B$. Then $B-\mathcal{V}$ is open and $\{K \in \Phi \mid K \cap \mathcal{V} \neq \varnothing\}=\Phi-\{K \in \Phi \mid K \subset B-\mathcal{V}\}$. Hence $i^{-1}[\{K \in \Phi \mid K \cap \mathcal{V} \neq \varnothing\}]=A-i^{-1}[\{K \in \Phi \mid K \subset B-\mathcal{V}\}]$ which is a $G_{\delta}$-subset in light of (a).

(c) Finally, consider $i^{-1}[\{\mathcal{K} \in \Phi \mid \mathcal{K} \cap \mathcal{U} \neq \varnothing\}]$, where $\mathcal{U}$ is an arbitrary open subset of $B$. There is a sequence $\left\langle\mathcal{V}_{n}\right\rangle$ of compact, open subsets of $B$ having $U=$ $\bigcup\left\{\mathcal{V}_{n} \mid n \geq 1\right\}$ so that $i^{-1}[\{\mathcal{K} \in \Phi \mid \mathcal{K} \cap \mathcal{U} \neq \varnothing\}]=\bigcup\left\{i^{-1}\left[\left\{\mathcal{K} \in \Phi \mid \mathcal{K} \cap \mathcal{V}_{n} \neq \varnothing\right\}\right] \mid n \geq\right.$ $1\}$ which is a $G_{\delta \sigma^{-}}$set in $\mathcal{A}$ because of (b).

(d) Since sets of the form $\{K \in \Phi \mid \mathcal{K} \subset \mathcal{U}\}$ and $\{K \in \Phi \mid \mathcal{K} \cap \mathcal{U} \neq \varnothing\}$ form a subbase for the separable metric space $\Phi$, it follows that $i$ is a Borel mapping of class 2 .

3.6 LEMMA. With $\Phi_{S}$ as defined in $3.4, \Phi_{S}$ is a Borel subset of $\Phi$ whose additive class is $\alpha$ (= the additive class of $S)$.

PROOF. For $n \geq 1$, define $\theta_{n}:\left(2^{\omega}\right)^{n} \rightarrow \Phi$ by $\theta_{n}\left(x_{1}, x_{2}, \ldots, x_{n}\right)=\left\{B_{x_{1}}, B_{x_{2}}\right.$, $\left.\ldots, B_{x_{n}}\right\}$. Then $\theta_{n}$ is continuous. Let $G_{n}=\left\{\left(x_{1}, \ldots, x_{n}\right) \in\left(2^{\omega}\right)^{n} \mid x_{j} \neq x_{k}\right.$ whenever $1 \leq j<k \leq n\}$. Then $G_{n}$ is open in $\left(2^{\omega}\right)^{n}$ and given $\left(x_{1}, \ldots, x_{n}\right) \in G_{n}$ there is an open neighborhood $N$ of $\left(x_{1}, \ldots, x_{n}\right)$ in $G_{n}$ and an open neighborhood $\Phi^{\prime}$ of $\theta_{n}\left(x_{1}, \ldots, x_{n}\right)$ in $\Phi$ such that $\theta_{n}$ maps $N$ homeomorphically onto $\Phi^{\prime} \cap \Phi_{n}$. (We say that $\theta_{n}$ is a local homeomorphism from $G_{n}$ onto $\Phi_{n}$.)

Now consider the subspace $S$ of $2^{\omega}$. Clearly $\theta_{n}\left[G_{n} \cap S^{n}\right]=\Phi_{n} \cap \Phi_{S}$ so $\theta_{n}$ is a local homeomorphism from $G_{n} \cap S^{n}$ onto $\Phi_{n} \cap \Phi_{S}$. Because $S$ is of additive class $\alpha$, so is $S^{n}\left[\mathbf{K}\right.$, p. 346]. Hence so is $G_{n} \cap S^{n}$ as is each relatively open subset of $G_{n} \cap S^{n}$. 
(Recall that since $\alpha \geq 1$, each open subset of $G_{n}$ is of additive class $\alpha$.) Therefore, the metric space $\Phi_{S} \cap \Phi_{n}$ admits an open cover by sets of additive class $\alpha$ so that $\Phi_{S} \cap \Phi_{n}$ has additive class $\alpha\left[\mathbf{K}\right.$, p. 358]. Because $\Phi_{S}=\{\varnothing\} \cup\left(\bigcup\left\{\Phi_{S} \cap \Phi_{n} \mid n \geq 1\right\}\right)$, $\Phi_{S}$ also has additive class $\alpha$, as claimed.

3.7 Lemma. Let $D=\{A \in \mathcal{P}(T) \mid$ some $P \in p$ has $P \cap A=\varnothing\}$. Then $D$ is of additive class $2+\alpha$.

Proof. With $i$ as in 3.5 , we claim that $D=i^{-1}\left[\Phi_{S}\right]$. For let $A \in D$. Choose $P \in p$ with $P \cap A=\varnothing$. Then $P$ contains some set $T-\left(B_{x_{1}} \cup \cdots \cup B_{x_{n}} \cup F\right)$, where $x_{j} \in S$, so $A \subset B_{x_{1}} \cup \cdots \cup B_{x_{n}} \cup F$. Hence $A \in \mathcal{A}$ so that $i(A)$ is defined. As noted in the proof of 3.5 , since $A \subset B_{x_{1}} \cup \cdots \cup B_{x_{n}} \cup F, i(A) \subset\left\{B_{x_{1}}, \ldots, B_{x_{n}}\right\}$ showing that $i(A) \in \Phi_{S}$. Conversely, suppose $A \in i^{-1}\left[\Phi_{S}\right]$. Then either there are points $x_{1}, \ldots, x_{n} \in S$ with $i(A)=\left\{B_{x_{1}}, \ldots, B_{x_{n}}\right\}$ or else $i(A)=\varnothing$ in which case $A$ is finite. Consider the first possibility. If the set $A-\left(B_{x_{1}} \cup \cdots \cup B_{x_{n}}\right)$ were infinite, some other branch of $T$ would have an infinite intersection with $A$ which is impossible, so the set $F=A-\left(B_{x_{1}} \cup \cdots \cup B_{x_{n}}\right)$ is finite and we have $A \subset B_{x_{1}} \cup \cdots \cup B_{x_{n}} \cup F$, so that $A$ is disjoint from $T-\left(B_{x_{1}} \cup \cdots \cup B_{x_{n}} \cup F\right)$ which belongs to the filter $p$ so that $A \in D$. The case where $A$ is finite is easy because then the set $P_{0}=T-A$ belongs to $p$ so that $A \in D$.

Because $i$ is a Borel map of class 2 and because by 3.6 the set $\Phi_{S}$ has additive class $\alpha$ (where $\alpha$ is the additive class of $S$ ), $i^{-1}\left[\Phi_{S}\right]$ has additive class $2+\alpha$, as claimed.

4. The projective hierarchy. Recall the definition of the projective classes in a complete separable metric space $Z[\mathbf{K}$, Chapter $3, \S 38]$ :

$$
\begin{aligned}
& \mathcal{L}_{0}(Z)=\{A \mid A \text { is a Borel subset of } Z\}, \\
& \mathcal{L}_{n+1}(Z)=\left\{\begin{array}{l}
\left\{f[A] \mid A \in \mathcal{L}_{n}(Z) \text { and } f: A \rightarrow Z \text { is continuous }\right\} \\
\left\{Z-A \mid A \in \mathcal{L}_{n}(Z)\right\} \text { if } n \text { is even, }
\end{array}\right.
\end{aligned}
$$

Thus, $\mathcal{L}_{1}(Z)$ is the family of analytic sets in $Z, \mathcal{L}_{2}(Z)$ is the family of co-analytic sets in $Z$, etc. The techniques of $\S \S 2$ and 3 can be used to prove an analogue of 3.1 for projective sets. In our proof we will invoke theorems which are ordinarily stated for mappings into complete metric spaces [K, $\S 38$, III, Propositions 2 and 5, and VII, Theorem 1], applying those results to mappings into the $\sigma$-compact metric space $\Phi$ defined in 3.4. Extending the proofs given in $[\mathbf{K}]$ to cover this situation is easily done.

4.1 TheOREM. Suppose $S \in \mathcal{L}_{r}\left(2^{\omega}\right)$ for some $r \geq 1$. Let $\Sigma=\Sigma_{S}$. Then $C_{\pi}(\Sigma) \in \mathcal{L}_{r}\left(\mathbf{R}^{\Sigma}\right)$. Furthermore, if $S \notin \mathcal{L}_{r-1}\left(2^{\omega}\right)$, then $C_{\pi}(\Sigma) \notin \mathcal{L}_{r-1}\left(\mathbf{R}^{\Sigma}\right)$.

Proof. Define $\psi_{m}: \mathbf{R}^{\Sigma} \rightarrow \mathcal{P}(T)$ and $D \subset \mathcal{P}(T)$ as in 3.1. Suppose we know that $D \in \mathcal{L}_{r}(\mathcal{P}(T))$. Then by $[\mathbf{K}, \S 38$, III, Proposition 5$], \psi_{m}^{-1}[D] \in \mathcal{L}_{r}\left(\mathbf{R}^{\Sigma}\right)$ for each $m$ so that by $\left[\mathbf{K}, \S 38\right.$, III, Proposition 3] we would have $C_{\pi}(\Sigma)=\bigcap_{m=1}^{\infty} \psi_{m}^{-1}[D] \in \mathcal{L}_{r}\left(\mathbf{R}^{\Sigma}\right)$ as claimed. Thus it will be enough to show that $D \in \mathcal{L}_{r}(\mathcal{P}(T))$.

To prove that $D \in \mathcal{L}_{r}(P(T))$, we define the $\sigma$-compact set $A \subset P(T)$ as in 3.3, the $\sigma$-compact metric space $\Phi$ as in 3.4, the Borel measurable mapping $i: A \rightarrow \Phi$ as in (3.5), and the set $\Phi_{S}$ as in 3.4. As in the proof of 3.7, $D=A \cap i^{-1}\left[\Phi_{S}\right]$. If we knew that $\Phi_{S} \in \mathcal{L}_{r}(\Phi)$, it would follow from [K, $\S 38$, III, Proposition 5] that $i^{-1}\left[\Phi_{S}\right] \in \mathcal{L}_{r}(\mathcal{A})$. Since $\mathcal{A}$ is $\sigma$-compact and hence in $\mathcal{L}_{r}(P(T))$, it would follow 
that $D \in \mathcal{L}_{r}(\mathcal{P}(T))[\mathbf{K}, \S 38$, III, Proposition 2]. Therefore it will be enough to show that $\Phi_{S} \in \mathcal{L}_{r}(\Phi)$. Define function $\theta_{n}:\left(2^{\omega}\right)^{n} \rightarrow \Phi$ as in 3.6. According to $\left[\mathbf{K}, \S 38\right.$, III, Proposition 1], $S^{n} \in \mathcal{L}_{r}\left(\left(2^{\omega}\right)^{n}\right)$. Because each open subset $H$ of $\left(2^{\omega}\right)^{n}$ also belongs to $\mathcal{L}_{r}\left(\left(2^{\omega}\right)^{n}\right)$ we see that $H \cap G_{n} \cap S^{n} \in \mathcal{L}_{r}\left(\left(2^{\omega}\right)^{n}\right)$ whenever $H$ is open in $\left(2^{\omega}\right)^{n}$. But $\theta_{n}$ is known to be a local homeomorphism of $G_{n} \cap S^{n}$ onto the separable metric space $\Phi_{S} \cap \Phi_{n}$ so there is a sequence $H_{1}, H_{2}, \ldots$ of subsets of $G_{n}$ such that for each $k, \theta_{n}$ maps $H_{k} \cap G_{n} \cap S^{n}$ homeomorphically onto a relatively open subset of $\Phi_{n} \cap \Phi_{S}$ and such that $\Phi_{n} \cap \Phi_{S}=\bigcup\left\{\theta_{n}\left[H_{k} \cap G_{n} \cap \dot{S}^{n}\right] \mid k \geq 1\right\}$. Because $H_{k} \cap G_{n} \cap S^{n} \in \mathcal{L}_{r}\left(\left(2^{\omega}\right)^{n}\right)$ for each $k$, it follows from [K, $\S 38$, VII, Theorem 1] that $\theta_{n}\left[H_{k} \cap G_{n} \cap S^{n}\right] \in \mathcal{L}_{r}(\Phi)$. But then $\Phi_{n} \cap \Phi_{S}$, being a countable union of members of $\mathcal{L}_{r}(\Phi)$, also belongs to $\mathcal{L}_{r}(\Phi)$. For the same reason, the set $\Phi_{S}=\bigcup\left\{\Phi_{S} \cap \Phi_{n} \mid n \geq 1\right\}$ also belongs to $\mathcal{L}_{r}(\Phi)$ as claimed.

Finally suppose $S \notin \mathcal{L}_{r-1}\left(2^{\omega}\right)$. According to 2.1 , there is a (relatively) closed subspace $S^{*}$ of $C_{\pi}(\Sigma)$ which is homeomorphic to $S$. Then $S^{*}=C_{\pi}(\Sigma) \cap D$, where $D$ is some closed subset in $\mathbf{R}^{\Sigma}$. If $C_{\pi}(\Sigma) \in \mathcal{L}_{r-1}\left(\mathbf{R}^{\Sigma}\right)$, then $S^{*}=C_{\pi}(\Sigma) \cap D$ would also belong to $\mathcal{L}_{r-1}\left(\mathbf{R}^{\Sigma}\right)$. According to [K, $\S 38$, VII, Theorem 1], we would then have $S \in \mathcal{L}_{r-1}\left(2^{\omega}\right)$ because $S$ is homeomorphic to $S^{*}$, which is impossible.

4.2 COROLlaRY. For each $n \geq 1$ there is a countable regular space $X_{n}$ such that $C_{\pi}\left(X_{n}\right) \in \mathcal{L}_{n}\left(\mathbf{R}^{X_{n}}\right)-\mathcal{L}_{n-1}\left(\mathbf{R}^{X_{n}}\right)$ and there is a countable regular space $Y$ such that $C_{\pi}(Y) \notin \bigcup\left\{\mathcal{L}_{n}\left(\mathbf{R}^{Y}\right) \mid n \geq 1\right\}$.

Proof. Fix $n$. By $\left[\mathbf{K}, \S 38\right.$, VI, Theorem 1] there is a set $S_{n} \subset 2^{\omega}$ having $S_{n} \in \mathcal{L}_{n}\left(2^{\omega}\right)-\mathcal{L}_{n-1}\left(2^{\omega}\right)$. Let $X_{n}=\Sigma_{S_{n}}$. To obtain the space $Y$, choose any $S \subset 2^{\omega}$ with $S \notin \bigcup\left\{\mathcal{L}_{n}\left(2^{\omega}\right) \mid n \geq 1\right\}\left[\mathbf{K}, \S 38\right.$, VI, Remark 1] and let $Y=\Sigma_{S}$. Because $C_{\pi}(Y)$ contains a closed subset homeomorphic to $S, C_{\pi}(Y) \notin \bigcup\left\{\mathcal{L}_{n}\left(\mathbf{R}^{Y}\right) \mid n \geq 1\right\}$.

5. Baire category and Baire Property subsets of $\mathbf{R}^{X}$. For any space $Z$, $B P(Z)$ is the $\sigma$-algebra generated by the open sets and the first category subsets

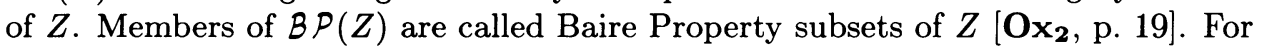
a space $X$ with a unique limit point (such as the spaces $\Sigma_{S}$ for $S \subset 2^{\omega}$ constructed in $\S 1$ ) it is easy to characterize which function spaces $C_{\pi}(X)$ belong to $B P\left(\mathbf{R}^{X}\right)$.

5.1 THEOREM. Suppose $X$ is a countable space with a unique limit point $\infty$ and let $p$ be the trace on $X-\{\infty\}$ of the neighborhod filter of $\infty$. Then the following are equivalent:

(a) $C_{\pi}(X)$ is a first category subset of $\mathbf{R}^{X}$;

(b) $C_{\pi}(X) \in B P\left(\mathbf{R}^{X}\right)$;

(c) there is an array

$$
\begin{array}{llll}
A(1,1) & A(1,2) & A(1,3) & \ldots \\
A(2,1) & A(2,2) & A(2,3) & \ldots \\
A(3,1) & A(3,2) & A(3,3) & \ldots
\end{array}
$$

satisfying

(i) each $A(m, n)$ is a finite subset of $X-\{\infty\}$;

(ii) each row $A(m, 1), A(m, 2), A(m, 3),, \ldots$ is a pairwise disjoint sequence;

(iii) for every sequence $k(1), k(2), \ldots$ and every $U \in p, U \cap(\bigcup\{A(m, k(m)) \mid m \geq$ $1\}) \neq \varnothing$. 
Proof. The equivalence of (a) and (c) follows from [LM, Theorems 6.3 and 5.1] and obviously (a) implies (b). We prove that (b) implies (a). Suppose $C_{\pi}(X) \in$ $B P\left(\mathbf{R}^{X}\right)$. To simplify notation, we will identify the countably many isolated points of $X$ with elements of $\omega$ and we will write $X=\omega \cup\{\infty\}$. Define a function $\nu: \mathbf{R}^{X} \rightarrow \mathbf{R}^{\omega} \times \mathbf{R}$ by the rule that $\nu(f)=\left(f^{*}, f(\infty)\right)$, where $f^{*} \in \mathbf{R}^{\omega}$ is given by $f^{*}(n)=f(n)-f(\infty)$. Then $\nu$ is a homeomorphism of $\mathbf{R}^{X}$ onto $\mathbf{R}^{\omega} \times \mathbf{R}$ and $\nu\left[C_{\pi}(X)\right]=C_{0} \times \mathbf{R}$, where $C_{0}=\left\{g \in \mathbf{R}^{\omega} \mid\right.$ for each $\varepsilon>0$ there is a neighborhood $U$ of $\infty$ having $g[U \cap \omega] \subset]-\varepsilon, \varepsilon[\}$. Since $C_{\pi}(X) \in B P\left(\mathbf{R}^{X}\right), C_{0} \times \mathbf{R} \in B P\left(\mathbf{R}^{\omega} \times \mathbf{R}\right)$.

It is easily seen that $C_{0}$ is a tailset in $\mathbf{R}^{\omega}$, i.e. that if $g \in C_{0}$ and if the equality $h(n)=g(n)$ holds except for finitely many values of $n$, then $h \in C_{0}$. We now need a slight variation of a result due to Oxtoby $\left[\mathbf{O} \mathbf{x}_{\mathbf{1}}\right]$; the proof is only trivially different from Oxtoby's argument.

5.2 Lemma. Let $C$ be a tailset in $\mathbf{R}^{\omega}$ and suppose that $C \times \mathbf{R} \in B P\left(\mathbf{R}^{\omega} \times \mathbf{R}\right)$. Then either $C \times \mathbf{R}$ is a first category subset of $\mathbf{R}^{\omega} \times \mathbf{R}$ or else $C \times \mathbf{R}$ contains a dense $G_{\delta}$-subset of $\mathbf{R}^{\omega} \times \mathbf{R}$.

Given 5.2, either $C_{0} \times \mathbf{R}$ is a first category subset of $\mathbf{R}^{\omega} \times \mathbf{R}$, in which case $C_{\pi}[X]$ is also a first category subset of $\mathbf{R}^{X}$, or else $C_{0} \times \mathbf{R}$ contains a dense $G_{\delta}$-subset of $\mathbf{R}^{\omega} \times \mathbf{R}$, in which case $C_{\pi}(X)$ contains a dense $G_{\delta}$ in $\mathbf{R}^{X}$. But the latter situation occurs if and only if $X$ is a discrete space [DGLvM, Theorem 1] so that $C_{\pi}(X)$ must be a first category subset of $\mathbf{R}^{X}$, as claimed.

5.3 REMARK. The reason for creating a variant of Oxtoby's theorem as in 5.2 is that one cannot deduce $C_{0} \in B P\left(\mathbf{R}^{\omega}\right)$ from $C_{0} \times \mathbf{R} \in B P\left(\mathbf{R}^{\omega} \times \mathbf{R}\right)$.

5.4 CoRollary. For each $S \subset 2^{\omega}$, the function space $C_{\pi}\left(\Sigma_{S}\right)$ is a first category subset of $\mathbf{R}^{\Sigma_{s}}$.

Proof. We define an array $A(m, n)$ as follows using the tree $T=\bigcup_{1}^{\infty} T_{n}$;

(i) $A(1, n)=T_{n}$ for $n \geq 1$;

(ii) $A(2,1)=T_{1} \cup T_{2}, A(2,2)=T_{3} \cup T_{4}, A(2,3)=T_{5} \cup T_{6}, \ldots$;

(iii) in general, $A(m, n)=T_{(n-1) m+1} \cup \cdots \cup T_{n m}$.

Obviously each $A(m, n)$ is finite and because the sets $T_{1}, T_{2}, \ldots$ are pairwise disjoint, each row $A(m, 1), A(m, 2), \ldots$ of the array is pairwise disjoint. Suppose $k(1), k(2), \ldots$ is a sequence of positive integers and suppose $U=T-\left(B_{x_{1}} \cup \cdots \cup B_{x_{n}} \cup\right.$ $F)$, where $x_{i} \in S$ and $F$ is a finite subset of $T$. If $\varnothing=U \cap(\bigcup\{A(m, k(m)) \mid m \geq 1\})$, then $\bigcup\{A(m, k(m)) \mid m \geq 1\} \subset B_{x_{1}} \cup B_{x_{2}} \cup \cdots \cup B_{x_{n}} \cup F$. Observe that for a fixed level $T_{j}$ of the tree $T, \operatorname{card}\left(B_{x_{i}} \cap T_{n}\right)=1$ so that $\operatorname{card}\left(T_{j} \cap\left(B_{x_{1}} \cup \cdots \cup B_{x_{n}} \cup F\right)\right) \leq$ $n+\operatorname{card}(F)$. Choose $m>n+\operatorname{card}(F)$. Then the set $A(m, k(m))$ contains a level $T_{j}$ of $T$ where $\operatorname{card}\left(T_{j}\right) \geq 2^{m}$ so that $T_{j} \cap\left(B_{x_{1}} \cup \cdots \cup B_{x_{n}} \cup F\right)$ must have cardinality greater than $n+\operatorname{card}(F)$, contrary to our observation above.

In closing let us give one more example of a countable regular space $X$ with a unique isolated point $\infty$ which has a "bad" function space. Unlike the examples so far, $C_{\pi}(X)$ is a second category subset of $\mathbf{R}^{X}$.

5.5 EXAMPLE. Let $p$ be a free ultrafilter on $\omega$ and topologize the set $X=\omega \cup\{\infty\}$ by isolating all points of $\omega$ and by using all sets of the form $\{\infty\} \cup U$, where $U \in p$, as neighborhoods of $\infty$. Then $C_{\pi}(X)$ is a second category subset of $\mathbf{R}^{X}$ and $C_{\pi}(X) \notin \mathcal{L}_{1}\left(\mathbf{R}^{X}\right) \cup \mathcal{L}_{2}\left(\mathbf{R}^{X}\right)$.

PROOF. That $C_{\pi}(X)$ is a second category subset of $\mathbf{R}^{X}$ follows from the equivalence of (a) and (c) in 5.1 (cf. [LM, 5.1 and 6.3] for details). Suppose 
$C_{\pi}(X) \in \mathcal{L}_{n}\left(\mathbf{R}^{X}\right)$, where $n \in\{1,2\}$. Define $j: 2^{\omega} \rightarrow \mathbf{R}^{X}$ by the rule that if $f \in 2^{\omega}$ then $j(f)=\hat{f} \in \mathbf{R}^{X}$ where $\hat{f}$ is given by

$$
\hat{f}(x)= \begin{cases}f(x) & \text { if } x \in \omega, \\ 1 & \text { if } x=\infty .\end{cases}
$$

Then $j$ is continuous so that by $\left[\mathbf{K}, \S 38\right.$, III, Proposition 2], $j^{-1}\left[C_{\pi}(X)\right] \in \mathcal{L}_{n}\left(2^{\omega}\right)$. Hence $j^{-1}\left[C_{\pi}(X)\right]$ is a measurable subset of $2^{\omega}$ (with respect to product measure $\mu$ ) because all analytic and co-analytic subsets of $2^{\omega}$ are measurable $[\mathbf{L}$, p. 243, Proposition 3.24]. But $j^{-1}\left[C_{\pi}(X)\right]=\left\{x \in 2^{\omega} \mid\right.$ for some $U \in p, x(n)=1$ for each $n \in U\}$ so that $j^{-1}\left[C_{\pi}(X)\right]$ is seen to be a tailset in $2^{\omega}$. Hence Kolmogorov's

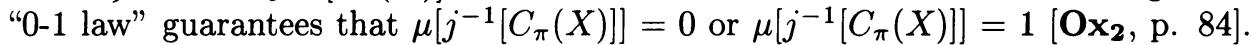
However, consider the function $J: 2^{\omega} \rightarrow 2^{\omega}$ given by $J(f)=f \oplus \overline{1}$, where $\overline{1} \in 2^{\omega}$ is constantly equal to 1 and $\oplus$ denotes coordinatewise addition modulo 2 , i.e., the usual group operation of $2^{\omega}$. Since $\mu$ is translation invariant, $J$ is a measure preserving transformation on $2^{\omega}$. Because $p$ is an ultrafilter, $J\left[j^{-1}\left[C_{\pi}(X)\right]\right]=$ $2^{\omega}-j^{-1}\left[C_{\pi}(X)\right]$ so that both $\mu\left[j^{-1}\left[C_{\pi}(X)\right]\right]=0$ and $\mu\left[j^{-1}\left[C_{\pi}(X)\right]\right]=1$ are impossible. Therefore $C_{\pi}(X) \notin \mathcal{L}_{0}\left(\mathbf{R}^{X}\right) \cup \mathcal{L}_{1}\left(\mathbf{R}^{X}\right) \cup \mathcal{L}_{2}\left(\mathbf{R}^{X}\right)$, as claimed.

\section{REFERENCES}

[DGLvM] J. Dijkstra, T. Grilliot, D. Lutzer and J. van Mill, Function spaces of low Borel complexity, Proc. Amer. Math. Soc. (to appear).

[E] R. Engelking, General topology, Polish Scientific Publishers, Warsaw, 1977.

[K] K. Kuratowski, Topology, vol. 1, Academic Press, New York, 1966.

$[$ KM] K. Kuratowski and A. Mostowski, Set theory with an introduction to descriptive theory, NorthHolland, Amsterdam, 1976.

[L] A. Levi, Basic set theory, Springer-Verlag, Berlin, 1979.

[LM] D. Lutzer and R. McCoy, Category in function spaces. I, Pacific J. Math. 90 (1980), 145-168.

[Ox 1 J. Oxtoby, Cartesian products of Baire spaces, Fund. Math. 49 (1961), 157-166.

$\left[\mathbf{O x}_{2}\right]$, Measure and category, Graduate Texts in Math., vol. 2, Springer-Verlag, New York, 1971.

Department of Mathematics, Miami University, OXford, OHio 45056

VRiJe Universiteit, Amsterdam, The Netherlands

Mathematics Institute, University of Warsaw, Warsaw, Poland 